Unfallchirurg 2019 · 122:4

https://doi.org/10.1007/s00113-018-0592-6

Online publiziert: 7. Dezember 2018

(c) Springer Medizin Verlag $\mathrm{GmbH}$, ein Teil von

Springer Nature 2018

\title{
Der Unfallchirurg dankt den Gutachtern 2018
}

Die Schriftleitung von Der Unfallchirurg dankt den Herausgebern der Zeitschrift und insbesondere den Herausgebern der Leitthemen sowie allen weiteren Experten und Expertinnen für ihre Unterstützung als wissenschaftliche Gutachter im Jahr 2018. Durch ihren Einsatz garantieren sie im Rahmen des Peer-Review-Verfahrens der eingereichten Manuskripte die hohe Qualität und klinische Relevanz von Der Unfallchirurg.

Michael Amlang, Dresden Hermann Bail, Nürnberg Arne Berner, Berlin Christopher Bliemel, Marburg Viktoria Bogner, München Johannes Bogner, München Sepp Braun, Innsbruck Stefan Buchmann, München Volker Bühren, Murnau Ulrich Chiellino, München Moritz Crönlein, München Ulf Culemann, Celle Michael Ehrenfeld, München Christian Ehrnthaller, München Ingo Flesch, Tübingen Stefan Förch, Augsburg Karl-Heinz Frosch, Hamburg Thomas Fuchs, Berlin Bernd Füchtmeier, Regensburg Julian Fürmetz, München Jens Geerling, Hannover Markus Gesslein, Nürnberg Christoph Haag, Lörrach Florian Haasters, München Michael Hackl, Köln Christine Hagen, München Bernd Hartmann, Berlin Jörg Hausdorf, München Paul Heidekrüger, Regensburg Tobias Helfen, München Stefan Hinterwimmer, München Reinhard Hoffmann, Frankfurt Michael Hoffmann, Neustadt Alexander Hoffmann, Kaiserslautern Robert Hube, München Stefan Huber-Wagner, München Martin Jaeger, Freiburg Karl-Georg Kanz, München
Holger Keil, Ludwigshafen Chlodwig Kirchhoff, München Till Klatte, Hamburg

Kaj Klaue, Lugano Matthias Knobe, Aachen Andreas Lenich, München Philipp Lichte, Aachen Cornelia Löffel, München David Merschin, Berlin Thomas Neubauer, Horn Carl Neuerburg, München Ludwig Ney, München Udo Obertacke, Mannheim Sabine Ochman, Münster Hans-Georg Palm, Ulm Dominik Pförringer, München Paul Puchwein, Graz Carsten Riemer, Minden Götz Röderer, Ulm Tim Rose, Leipzig Steffen Ruchholtz, Marburg Marcus Rudolph, Mannheim Thomas Ruffing, Kaiserslautern Markus Scheibel, Zürich Tobias Schiergens, München Benedikt Schliemann, Münster Arndt Peter Schulz, Kiel Sebastian Siebenlist, München Matthias Spalteholz, Leipzig Michael Spannagl, München Almut Tempka, Berlin Helen Vester, München Elias Volkmer, München Kilian Wegmann, Köln Marcel Winkelmann, Hannover Florian Wolf, München Christian Zeckey, München 